\title{
Tratamento cirúrgico de divertículo de Zenker: relato de caso
}

\author{
Surgical treatment of Zenker's diverticulum: case report
}

Tratamiento quirúrgico del divertículo de Zenker: reporte de caso

Isabella Nacur Pinheiro ${ }^{1 *}$, Ademar Cândido de Oliveira², Gabriela Paiva Santos ${ }^{1}$, Guilherme Dumont Campos Paiva ${ }^{1}$, Huberth André Vieira Zuba², Felipe Jean de Souza Ferreira ${ }^{1}$, Luiza Nacur Pinheiro ${ }^{3}$, Renata Tiemi Moreira de Resende ${ }^{2}$, Rúbria Paiva Santos

\section{RESUMO}

Objetivo: Relatar o caso de uma paciente com diagnóstico de divertículo faringoesofágico, também conhecido como divertículo de Zenker, submetida a tratamento cirúrgico, abordando a técnica cirúrgica, o desfecho e a evolução da paciente ao longo do acompanhamento. Detalhamento de caso: Paciente de 45 anos, sexo feminino, com quadro de disfagia e odinofagia progressivas, associada a sensação de globus e corpo estranho na garganta, além de refluxo gastroesofágico. Realizados exames de imagem que evidenciaram o divertículo hipofaríngeo de cerca de $3 \mathrm{~cm}$, sendo submetida a cervicotomia esquerda longitudinal e posterior diverticulectomia. Abordamos também a evolução pós cirúrgica da paciente. Considerações finais: $O$ divertículo de Zenker é uma patologia adquirida rara, e por isso, muitos cirurgiões formados não tiveram contato ou não têm experiência com as opções terapêuticas disponíveis (reparo aberto por cervicotomia esquerda e reparo por via endoscópica). Portanto, é importante o estudo teórico de tais técnicas, permitindo um desfecho favorável e sem complicações, na maioria das vezes.

Palavras-chave: Zenker, Divertículo, Esôfago.

\begin{abstract}
Objective: To report the case of a patient diagnosed with pharyngoesophageal diverticulum, also known as Zenker's diverticulum, who underwent surgical treatment, addressing the surgical technique, the outcome and the evolution of the patient during the follow-up. Case details: A 45-year-old female patient with progressive dysphagia and odynophagia, associated with globus sensation and foreign body in the throat, in assition to gastroesophageal reflux. Imaging exams were performed that showed a hypopharyngeal diverticulum of about $3 \mathrm{~cm}$, being submitted to longitudinal left cervicotomy and later diverticulectomy. We also discuss the patient's post-surgical evolution. Final considerations: Zenker's diverticulum is a rare acquired pathology, and for this reason, many trained surgeons have not had contact or have no experience with the available therapeutic options (open repair by left cervicotomy and repair by endoscopic approach). Therefore, the theoretical study of such techniques is important, allowing for a favorable outcome without complications, in most cases.
\end{abstract}

Keywords: Zenker, Diverticulum, Esophagus.

\section{RESUMEN}

Objetivo: Informar el caso de un paciente diagnosticado de divertículo faringoesofágico, también conocido como divertículo de Zenker, que fue sometido a tratamiento quirúrgico, abordando la técnica quirúrgica, el resultado y la evolución del paciente durante el seguimiento. Detalles del caso: Paciente de 45 años con disfagia progresiva y odinofagia, asociada a sensación de globo y cuerpo extraño en la garganta, además de reflujo gastroesofágico. Se realizaron exámenes de imagen que mostraron un divertículo hipofaríngeo de unos

${ }^{1}$ Faculdade de Minas (FAMINAS-BH), Belo Horizonte - MG. *E-mail: bellanacur@hotmail.com

2 Santa Casa de Misericórdia de Belo Horizonte (SCMBH), Belo Horizonte-MG

${ }^{3}$ Faculdade de Saúde e Ecologia Humana (FASEH), Vespasiano - MG.

SUBMETIDO EM: 8/2021

ACEITO EM: 8/2021

PUBLICADO EM: 9/2021 
$3 \mathrm{~cm}$, siendo sometido a cervicotomía izquierda longitudinal y posteriormente diverticulectomía. También comentamos la evolución posquirúrgica del paciente. Consideraciones finales: El divertículo de Zenker es una patología adquirida poco frecuente, por lo que muchos cirujanos capacitados no han tenido contacto o no tienen experiencia con las opciones terapéuticas disponibles (reparación abierta por cervicotomía izquierda y reparación por abordaje endoscópico). Por tanto, el estudio teórico de dichas técnicas es importante, permitiendo un resultado favorable y sin complicaciones, en la mayoría de los casos.

Palabras clave: Zenker, Divertículo, Esófago.

\section{INTRODUÇÃO}

O divertículo de Zenker, também conhecido como faringoesofágico, nada mais é que uma manifestação sacular das camadas mucosa e submucosa em uma região de fraqueza do esôfago, sendo por isso chamado falso divertículo. É localizado entre os músculos tireofaringeo e cricofaríngeo, na região do triângulo de Killian (ESCALANTE ET, et al., 2019; FITCHAT NA, et al., 2019).

A grande maioria dos pacientes no início da doença são assintomáticos devido, principalmente, ao tamanho do divertículo. A fisiopatologia ainda não é bem clara, mas acredita-se que com o passar do tempo e com o aumento da pressão intraluminal no local de fraqueza, o mesmo aumenta de tamanho. Associado a esse quadro há uma contratura precoce principalmente do músculo cricofaríngeo causando dismotilidade esofágica. O crescimento do divertículo auxilia no aparecimento dos sintomas, nos quais podemos destacar a disfagia, tosse, halitose, regurgitação de conteúdo alimentar não digerido entre outros (CALEFFI MC, et al., 2020).

O diagnóstico é feito geralmente pela radiografia contrastada de esôfago, estômago e duodeno (REED), no qual se vê material radiopaco em região sacular formada no esôfago. A endoscopia pode ser realizada, apesar do risco de perfuração do divertículo de forma inadvertida no momento do exame (SANTOS VM, et al., 2019; ESCALANTE ET, et al., 2019). As complicações dessa doença são principalmente pneumonia aspirativa, obstrução esofágica e perfuração do divertículo (SANTOS VM, et al., 2019).

O tratamento basicamente leva em consideração além da repercussão clínica do paciente, a idade e a presença de comorbidades. Em pacientes jovens, hígidos ou que apresentem alterações na anatomia, se torna preferível a técnica cirúrgica por via aberta, uma vez que ela apresenta melhores resultados a longo prazo, mas por outro lado, possui maiores complicações durante o procedimento e imediatamente após. Já em pacientes que tenham um alto risco cirúrgico, a técnica endoscópica é a de escolha, desde que o cirurgião tenha conhecimento e experiência para executá-la. Atualmente a via endoscópica está cada vez mais sendo realizada, por ser considerada uma técnica menos invasiva (FUENTES E, 2016).

Apesar de ser uma patologia, em suma, benigna, é uma doença rara- ocorrência de 0,01\% a 0,11\%-e, por isso, muitos cirurgiões formados não tiveram contato ou não têm experiência com as opções terapêuticas disponíveis (NESHEIWAT Z e ANTUNES C, 2021). Dessa forma, visando esclarecer o processo cirúrgico, evolução e desfechos, abaixo observa-se um relato de caso sobre o divertículo em questão.

\section{DETALHAMENTO DO CASO}

O caso foi mediante a aprovação 4.931.108 pelo Comitê de Ética e Pesquisa (CEP). Paciente de 45 anos, sexo feminino, parda, natural e procedente de Minas Gerais. Apresentou-se ao ambulatório de Cirurgia Geral na região central de Minas Gerais, encaminhada pelo gastroenterologista, devido aos sintomas sugestivos de Divertículo de Zenker.

Relatou que há aproximadamente três anos apresentava disfagia e odinofagia progressiva, associada a sensação de globus e corpo estranho na garganta após a ingestão de alimentos sólidos, levando-a ao ato reflexo de pigarrear como tentativa para expulsar o conteúdo. Outra sintomatologia presente é o constante refluxo gastroesofágico, levando-a a assumir a posição de sentar para dormir. Com o passar do tempo outras sintomatologias passaram a incomodá-la, tais como como tosses constantes e halitose, as quais implicaram em inibição social. A paciente negou perda ponderal, febre, náuseas, vômitos e alteração no hábito intestinal. 
Negou história familiar de câncer esofágico assim como câncer de cabeça e pescoço. Diabética, em uso de Metformina (850 mg após o almoço), hipertensa em uso de Furosemida (40 mg pela manhã) e informou uso diário de Omeprazol (40 mg, duas vezes ao dia). Devido às comorbidades apresentadas, Diabetes Mellitus e Hipertensão Arterial, a paciente classifica-se como ASA II. A paciente apresentou todos os exames já realizados, ao longo dos anos, para avaliação do médico cirurgião responsável. Os laudos dos exames foram descritos a seguir.

Endoscopia digestiva alta (EDA), realizada no dia 08/04/2016: presença de sinais endoscópicos compatíveis com divertículo de Zenker, com cerca de $3 \mathrm{~cm}$ de extensão, localizado posteriormente ao esôfago cervical, com colo adjacente ao músculo cricofaríngeo. Sinais endoscópicos de esofagite não erosiva. Presença de hérnia hiatal por deslizamento de pequeno porte. Pangastrite endoscópica edematosa leve. Presença de pólipos gástricos sésseis no corpo e fundo, com as seguintes características: Arredondados, com superfície regular e discretamente avermelhada, medindo entre 2 e $6 \mathrm{~mm}$. Duodeno endoscopicamente normal até o início da segunda porção.

Para confirmação foi realizado, na mesma data, biópsias de antro e corpo para histopatologia e com o mesmo material foi realizado o teste da urease. Os resultados do anatomopatológico do antro-corpo e dos pólipos, realizados no dia em questão, foram respectivamente: Presença de gastrite crônica do antro e corpo, sem atividade, de etiologia não determinada morfologicamente. Quadro morfológico compatível com pólipo de glândula fúndica. A pesquisa para $\mathrm{H}$. pylori obteve resultado negativo.

No dia 14/06/2016 a paciente foi submetida ao exame REED digital. As características observadas no exame foram: tempo de esvaziamento esofágico longo; Presença de hérnia hiatal; Sinal de divertículo de Zenker; Presença de refluxo gastroesofágico grau III que atinge o terço superior do esôfago.

Uma nova EDA foi solicitada para a programação cirúrgica, devido ao quadro de disfagia alta associada a pirose, apresentado pela paciente. As conclusões obtidas pela EDA, realizada no dia 05/02/2019, foram: presença de sinais endoscópicos compatíveis com divertículo de Zenker, com cerca de $3 \mathrm{~cm}$ de extensão, localizado posteriormente ao esôfago cervical, com colo adjacente ao músculo cricofaríngeo; Sinais endoscópicos de Doença do Refluxo Gastroesofágico (DRGE) não erosiva; Hérnia hiatal por deslizamento de grande tamanho; Pangastrite endoscópica edematosa leve; Pólipos gástricos-sésseis, arredondados, com superfície regular e alaranjada- no corpo e fundo, medindo entre 2 a $10 \mathrm{~mm}$; Duodeno endoscopicamente normal, até o início da segunda porção (Figura $1 \mathrm{~A}-\mathbf{H}$ ).

Figura 1 - Endoscopia Digestiva Alta em 05/02/2019.
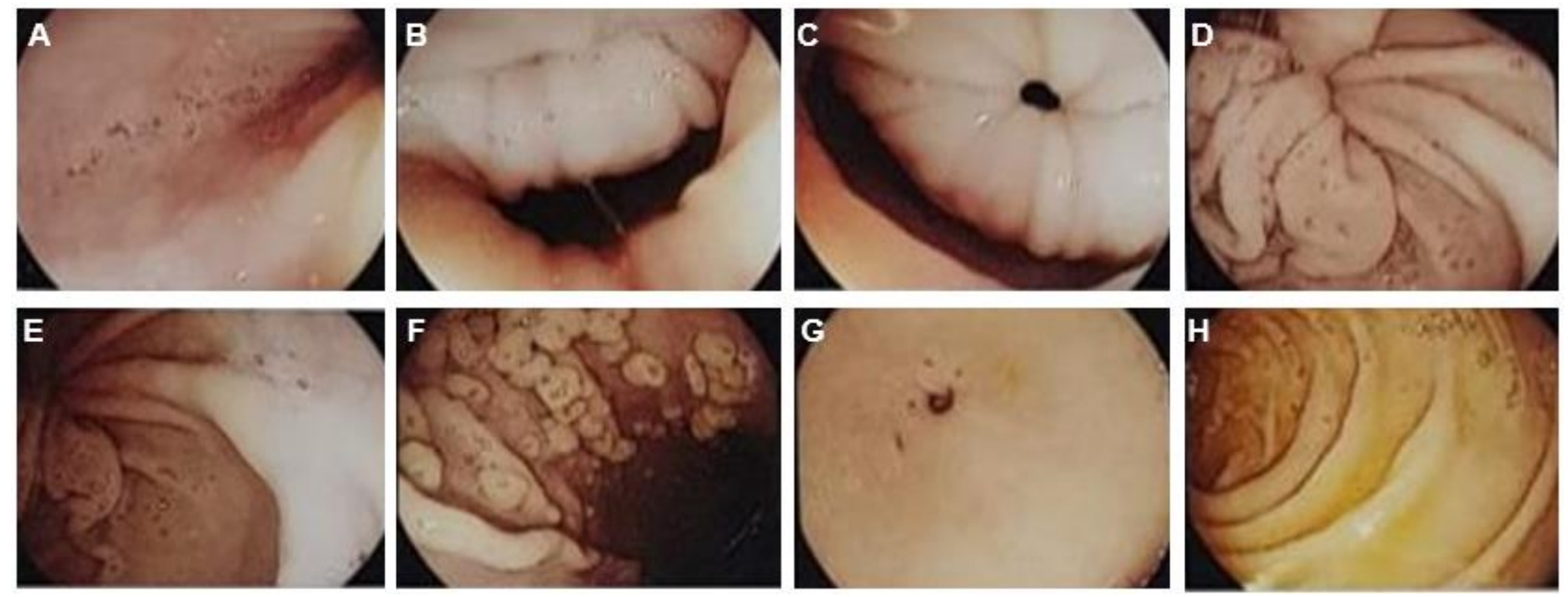

Fonte: Pinheiro IN, et al., 2021.

Uma nova EDA foi solicitada pelo médico assistente, com o objetivo de realizar a biópsia dos pólipos identificados no exame de imagem realizado posteriormente. Os achados endoscópicos, do exame realizado no dia 26/02/2019, foram semelhantes ao exame anterior e o resultado do anatomopatológico constou: Gastrite crônica do corpo e antro, sem atividade, de etiologia não determinada morfologicamente e morfológico compatível com pólipo de glândula fúngica (Figura 2 A-H). 
Figura 2 - Endoscopia Digestiva Alta em 26/02/2019.
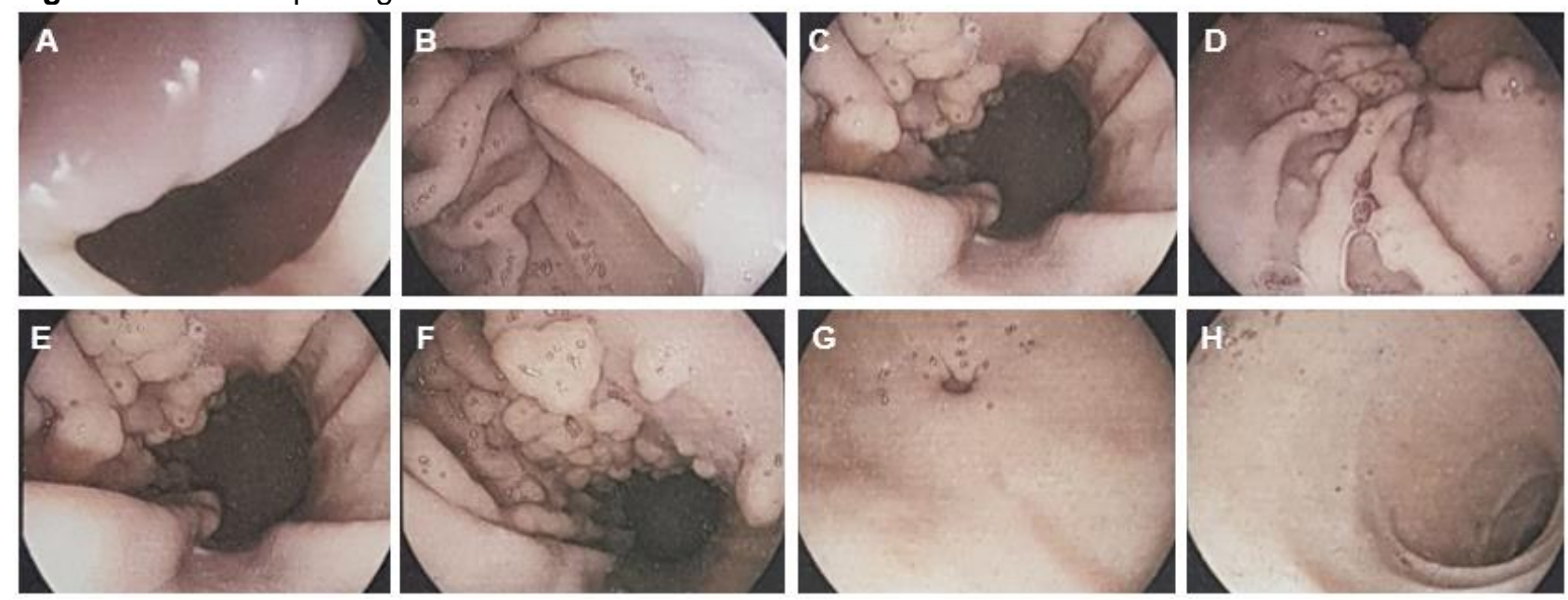

Fonte: Pinheiro IN, et al., 2021.

Após averiguação de todos os exames previamente realizados e da história clínica da paciente, foi confirmada a necessidade da realização do procedimento cirúrgico e feito o encaminhamento para avaliação pré-anestésica.

A diverticulectomia de Zenker foi realizada, no dia 19/02/2020, com os seguintes procedimentos: Com a paciente posicionada em decúbito dorsal, sob anestesia geral, foi realizada assepsia, antissepsia e colocação de campos estéreis. Posteriormente, fez-se cervicotomia esquerda longitudinal, na borda medial do músculo esternocleidomastóideo, com posterior dissecção por planos e o isolamento do divertículo. A partir desse momento, foi possível fazer a identificação do divertículo esofágico de cerca de $2,5 \mathrm{~cm}$, e a estrutura foi isolada após a passagem da sonda FOICHET.

Em seguida, foi feita a miotomia, a qual consiste em seccionar a musculatura do esfíncter cricofaríngeo, a partir do grampeamento da base, com granjeador linear de $80 \mathrm{~mm}$. Após, fez-se a diverticulectomia, com envio para estudo anatomopatológico da peça cirúrgica. Nos momentos finais da cirurgia, executou-se a revisão da hemostasia, deixando um dreno PENROSE número 1, na região inferior à tireoide. Por fim, houve fechamento por planos e realização de curativo (Figura 3, Figura 4A e 4B e Figura 5). Após o procedimento cirúrgico a paciente foi encaminhada para a enfermaria e recebeu alta no segundo dia de pós-operatório.

Figura 3 - Cervicotomia esquerda longitudinal, na borda medial do músculo esternocleidomastóideo, com posterior dissecção por planos.

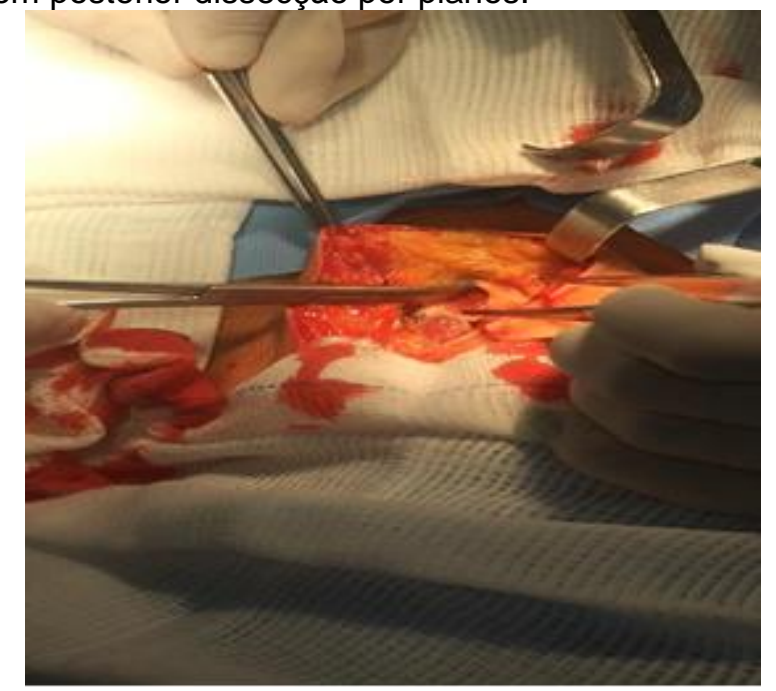

Fonte: Paiva RS, et al., 2021. 
Figura 4 - Identificação e isolamento do divertículo esofágico de cerca de $2,5 \mathrm{~cm}$ após a passagem da sonda FOICHET.

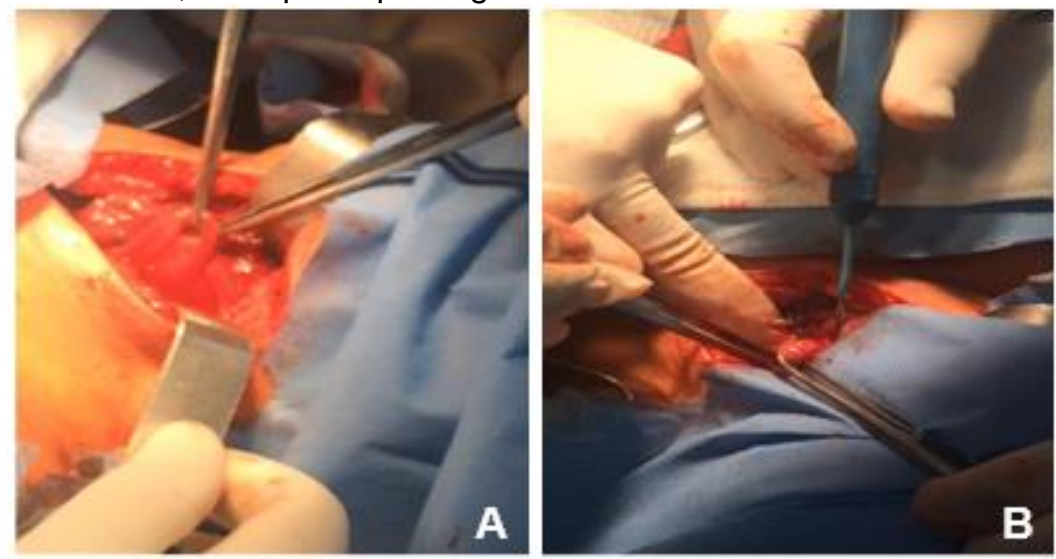

Fonte: Paiva RS, et al., 2021.

Figura 5 - Miotomia do esfíncter cricofaríngeo, a partir do grampeamento da base, com granjeador linear de $80 \mathrm{~mm}$.

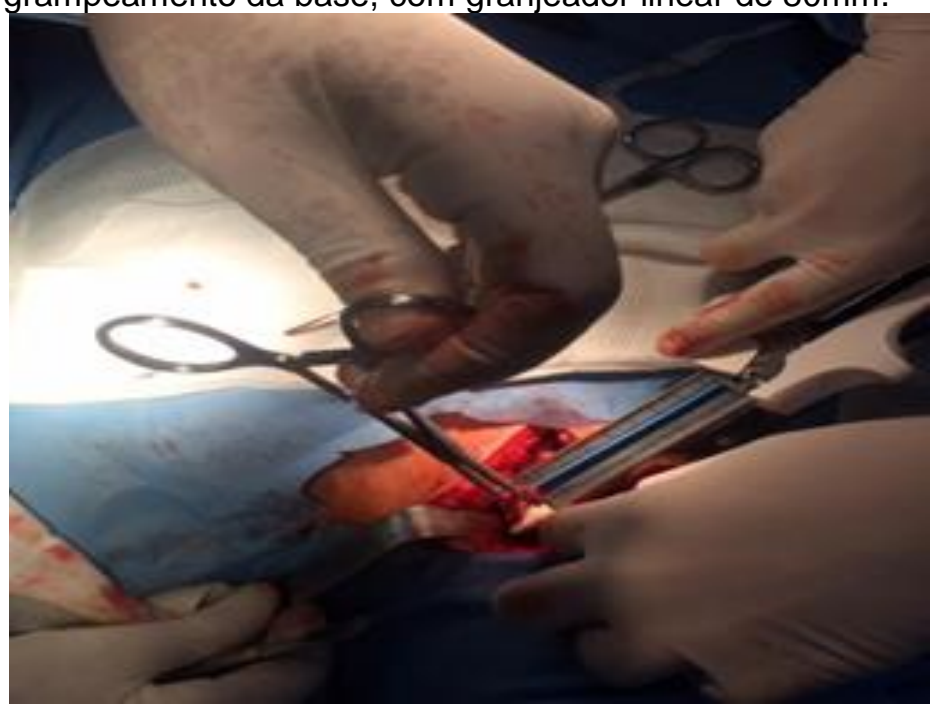

Fonte: Paiva RS, et al., 2021.

Figura 6 - Peça cirúrgica: Divertículo esofágico de cerca de $2,5 \mathrm{~cm}$.

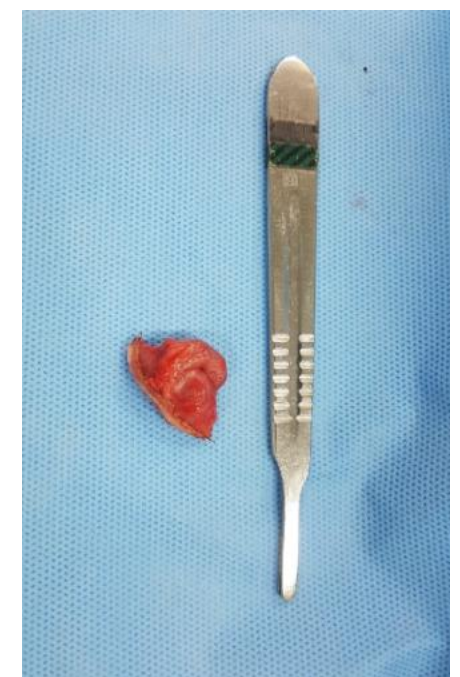

Fonte: Paiva RS, et al., 2021. 
Durante a internação a paciente apresentou boa recuperação, sem intercorrências e com ausência de febre, náuseas e vômitos, além de relato de diurese espontânea e evacuação, no primeiro e segundo dia pósoperatório, respectivamente. Além disso, a paciente deambulou sem dificuldades, manteve o índice glicêmico controlado durante todo o período de internação e os dados vitais permaneceram dentro da normalidade. A ferida operatória estava em bom aspecto, sem sinais de deiscência de sutura; o dreno cervical penrose se manteve bem-posicionado, com presença de secreção sanguinolenta em pequena quantidade. No primeiro dia pós-operatório foi prescrita dieta líquida completa, com progressão para dieta pastosa e branda -no segundo dia-, a qual houve boa aceitação, com relato de leve disfagia. $\mathrm{Na}$ alta, a paciente recebeu orientação para manter dieta branda e repouso por 15 dias, troca de curativo diariamente e retorno para avaliação com a equipe cirúrgica em duas semanas, munida do resultado do anatomopatológico.

A paciente retornou, duas semanas depois, no dia 06/03/2020, ao ambulatório, com o resultado do exame anatomopatológico, o qual acusou que a peça se tratava de divertículo de Zenker, sem nenhuma outra alteração, como displasia ou neoplasia evidente. Ela relatou que permanecia com leve disfagia ao ingerir alimentos sólidos e para deglutir comprimidos. Relatou boa aceitação para ingestão de alimentos pastosos e líquidos, assim como melhora do quadro de refluxo. Negou queixas álgicas ou alterações dos hábitos intestinais. Ao exame físico, evidenciou-se que a ferida operatória se apresentava em bom aspecto, sem sinais de deiscência e sem drenagem de secreção, com excelente cicatrização.

Como conduta foi instituído que a paciente retornasse em 30 dias e durante esse período optasse por ingerir alimentos de consistência líquida e pastosa. A mesma foi liberada para retornar às atividades laborais, assim como para realizar atividades físicas leves, sendo recomendado que a paciente reduzisse seu peso corporal, para um futuro planejamento de cirurgia para correção da hérnia hiatal.

\section{DISCUSSÃO}

Como já elucidado, o divertículo de Zenker é considerado um falso divertículo devido à manifestação sacular das camadas mucosa e submucosa entre os músculos tireofaríngeo e cricofaríngeo, na região do triângulo de Killian (LAW R, et al., 2014). A presença do divertículo geralmente desencadeia diversos sintomas que podem evoluir de meses há anos, até conclusão do diagnóstico, uma vez que o exame físico é frequentemente sem alterações, o que condiz com o caso citado (KOSTAKIS ID, et al., 2011). Os sintomas podem variar de disfagia (sintoma mais comum), tosse, halitose, regurgitação e engasgos, há disfonia, perda de peso e visível saculação em região acometida (LAW R, et al., 2014).

A fisiopatologia pode ser resumida da seguinte forma: Ocorre a protrusão da saculação, devido à fraqueza muscular local, o que leva ao acúmulo local de alimentos não digeridos (KOSTAKIS ID, et al., 2011). Quando comprime a parede esofagiana anterior, gera a disfagia (PINELAS A, 2015)

Quando não se faz o diagnóstico precoce, à medida que o divertículo se desenvolve a deglutição vai se tornando cada vez mais complicada, levando a perda de peso e desnutrição; outros sintomas supracitados também se tornam evidentes (ANDREIS EL, 2001). Além disso, as chances de aspiração pulmonar seguida de insuficiência respiratória, infecções respiratórias, broncoespasmo aumentam (PINELAS A, 2015). A paciente em questão não teve nenhuma dessas complicações mais graves citadas.

O que determina primariamente o tratamento para divertículo não é seu tamanho, mas sim, a presença ou não de sintomas (BONAVINA L, 2013). Divertículos pequenos e sintomáticos devem ser retirados; Divertículo grandes assintomáticos podem ser acompanhados clinicamente, e retirados na presença de sintomas, devido a estética, ou quando suspeita de carcinoma (achado raro); todavia, a abordagem expectante pode levar a cirurgias de urgência, que não são indicadas (PINELAS A, 2015; KOSTAKIS ID, et al., 2011).

Dessa forma, o tratamento cirúrgico eletivo deve ser considerado, uma vez que não há contraindicações absolutas para a cirurgia em questão (YUAN Y, et al., 2013). Para pacientes com idade avançada e que apresentam déficit nutricional ou complicações respiratórias devido ao divertículo é solicitado suporte adequado anterior à cirurgia (ANDREOLLO NA, et al., 2007). 
O divertículo pode ser retirado com realização de uma miotomia cricofaríngea (divertículo $<2 \mathrm{~cm}$ ), ou da miotomia acompanhada da diverticulectomia (divertículo $>2 \mathrm{~cm}$ ) sendo esta acompanhada ou não da diverticulectomia (YUAN Y, et al., 2013). No passado, a diverticulectomia era acompanhada da diverticulopexia para os de tamanho intermediário, para evitar a formação de fístulas, e consequentemente, anastomose esofagiana; ou em combinação com a sua inversão para os de menor tamanho (BONAVINA L, 2013; LAW R, et al., 2014; ANDREOLLO NA, et al., 2007).

As abordagens cirúrgicas podem ser de forma convencional (cervicotomia) ou endoscópica, de acordo com comorbidade, tamanho e recidiva do divertículo (PINELAS A, 2015). A cervicotomia é preconizada para divertículos $<2 \mathrm{~cm}$ ou volumosos $>6 \mathrm{~cm}$; uma vez que na abordagem endoscópica, divertículos muito pequenos e muito grandes não conseguem ser retirados completamente (VAN OJ, 2003). Em caso de falha da técnica endoscópica ou incapacidade de obtenção de uma exposição endoscópica adequadas, a cervicotomia também é a abordagem de escolha (PRISMAN E e GENDEN EM, 2013).

Quando na presença de comorbidades, e em divertículos de tamanho entre 2 e $6 \mathrm{~cm}$, a abordagem preconizada é a endoscópica, visto que é menos invasiva, sem necessidade de incisão (ANDREOLLO NA, et al., 2007). Dificuldades para hiperextensão do pescoço, abertura da cavidade bucal e obesidade podem inviabilizar a realização da técnica por via endoscópica (PRISMAN E e GENDEN EM, 2013). No caso em questão, foi optado por via aberta devido à experiência do cirurgião assistente.

A seguir, a técnica cirúrgica (cervicotomia) e endoscópica são explicadas: A cervicotomia é realizada com o paciente em decúbito dorsal, com pescoço estendido e rotacionado $45^{\circ}$ para a direita (WEISSBROD PA e MERATI AL, 2012). É feito o procedimento de colocação de sonda nasogástrica ou orogástrica (LAW R, et al., 2014). Posteriormente, é realizada a incisão longitudinal ao longo do bordo anterior do músculo esternocleidomastoideo (WEISSBROD PA e MERATI AL, 2012). Os tecidos são então dissecados, retirando as fibras musculares do cricofaríngeo para que seja possível visualizar o colo do divertículo (LAW R, et al., 2014). Isola-se o divertículo e remove-o (diverticulectomia) (LAW R, et al., 2014). Consecutivamente, é executada a miotomia completa do músculo cricofaríngeo com aproximadamente $3 \mathrm{~cm}$ direcionados ao esôfago distal (MARIETTE C, 2014). A sonda é removida e é realizado estudo radiológico contrastado com gastrografina, no dia seguinte; caso satisfatório, permite o início de dieta líquida oral (LAW R, et al., 2014).

Já a técnica endoscópica é feita com o paciente em decúbito dorsal e pescoço hiperestendido, sob anestesia geral (PINELAS A, 2015). Realizado divertículo-esofagotomia, passando o fibroscópio e expondo o septo entre esôfago e divertículo, removendo-o com sutura mecânica. Realizado estudo contrastado no primeiro dia de pós-operatório, quando sem alterações é permitido iniciar dieta oral líquida. Alta dentro de 24 horas após a cirurgia (PRISMAN E e GENDEN EM, 2013).

O divertículo de Zenker, descrito no relato de caso, apesar de ser uma patologia benigna pode se tornar extremamente incômoda para o paciente. Portanto, é imprescindível que mais estudos científicos sobre o tema sejam publicados, uma vez que o acesso ao conhecimento de técnicas cirúrgicas resolutivas, como as mencionadas, contribui grandemente para levar conforto e qualidade de vida para os pacientes acometidos. Além disso, como mencionado acima, a cirurgia possui excelentes resultados, com boa evolução pósoperatória e poucas intercorrências durante o ato cirúrgico.

\section{REFERÊNCIAS}

1. ANDREIS EL, et al. Divertículo de Zenker. Revista do Colégio Brasileiro de Cirurgiões, 2001; 28(4): 296-298.

2. ANDREOLLO NA, et al. Tratamento cirúrgico do divertículo de Zenker: Diverticulopexia versus Diverticulectomia. ABCD. Arquivos Brasileiros de Cirurgia Digestiva, 2007; 20(4): 245-249.

3. BONAVINA L. Surgical Management of Esophageal Diverticula, Chapter 30. In Shackelford's Surgery of the Alimentary Tract.: Elsevier Inc., 2013; 362-366.

4. CALEFFI MC, et al. Divertículo de Zenker: do diagnóstico ao tratamento. Divertículo de Zenker: do diagnóstico ao tratamento, 2020; 1-388.

5. FITCHAT NA, et al. Why do Zenker's diverticulae occur more often on the left than the right side? J Laryngol Otol., 2019; 133(6): 515-519. 
6. FUENTES EV. Tratamento atual para divertículo de Zenker. Rev Cubana Cir. 2016; 55(4): 343-348.

7. KOSTAKIS ID, et al. Zenker's diverticulum presented with chronic dry cough and recurrent pulmonary infections. "Laiko" General Hospital, Greece: Athens Medical Society, 2011; 28(5): 698-701.

8. LAW R, et al. Zenker's Diverticulum. In Clinical Gastroenterology and Hepatology; Division of Gastroenterology and Hepatology, Mayo Clinic, 2014; 28(5): 1-10.

9. MARIETTE C. Zenker's pharyngo-esophageal diverticulum: Diverticulectomy and diverticulopexy. Journal of Visceral Surgery, 2014; 151(2): 145-149.

10. NESHEIWAT Z, ANTUNES C. Zenker Diverticulum, StatPearls [Internet]. Treasure Island (FL): StatPearls Publishing, 2021.

11. PINELAS A. Divertículo de Zenker: Resultados da casuística da unidade de cirurgia digestiva do Centro Hospitalar do Porto. (Mestrado Integrado em Medicina), 2015.

12. PRISMAN E, GENDEN EM. Zenker Diverticulum, 2013; 46(6): 1101-1111.

13. VAN OJ. Pathogenesis and methods of treatment of Zenker's divericulum. Ann Otol Rhinol Laryngol, 2003; 112: 583593.

14. WEISSBROD PA, MERATI AL. Open surgery for Zenker's diverticulum. In Operative Techniques in Otolaryngology Head and Neck Surgery, 2012; 23(2): 137-143.

15. YUAN Y, et al. Surgical Treatment of Zenker's diverticulum. Dig Surg, 2013: 30(3): 214-225. 